\title{
Analysis method for Thomson scattering diagnostics in GAMMA 10/PDX
}

K. Ohta, M. Yoshikawa, R. Yasuhara, M. Chikatsu, Y. Shima, J. Kohagura, M. Sakamoto, Y. Nakasima, T. Imai, M. Ichimura, I. Yamada, H. Funaba, and T. Minami

Citation: Rev. Sci. Instrum. 87, 11E730 (2016); doi: 10.1063/1.4963161

View online: http://dx.doi.org/10.1063/1.4963161

View Table of Contents: http://aip.scitation.org/toc/rsi/87/11

Published by the American Institute of Physics

๑ SHIMADZU Powerful, Multi-functional UV-Vis-NIR and Excellence in Science FJis Spectjophotometers

Providing the utmost in sensitivity, accuracy and resolution for applications in materials characterization and science

- Photovoltaics

- Polymers

- Coatings

- Paints

Click here for accurate, cost-effective laboratory solutions
- DNA film structures

- Packaging materials

- Nanotechnology

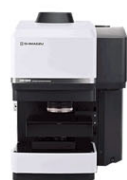

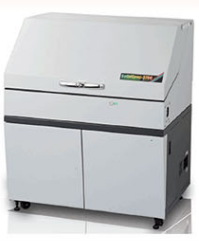




\title{
Analysis method for Thomson scattering diagnostics in GAMMA 10/PDX
}

\author{
K. Ohta,,${ }^{1, a)}$ M. Yoshikawa,${ }^{1}$ R. Yasuhara, ${ }^{2}$ M. Chikatsu, ${ }^{1}$ Y. Shima, ${ }^{1}$ J. Kohagura, ${ }^{1}$ \\ M. Sakamoto, ${ }^{1}$ Y. Nakasima,${ }^{1}$ T. Imai, ${ }^{1}$ M. Ichimura,${ }^{1}$ I. Yamada,${ }^{2}$ H. Funaba,${ }^{2}$ \\ and T. Minami ${ }^{3}$ \\ ${ }^{1}$ Plasma Research Center, University of Tsukuba, 1-1-1 Tennodai, Tsukuba, Ibaraki 305-8577, Japan \\ ${ }^{2}$ National Institute for Fusion Science, 322-6 Oroshi-cho, Toki, Gifu 509-5292, Japan \\ ${ }^{3}$ Institute of Advanced Energy, Kyoto University, Gokasho, Uji, Kyoto 611-0011, Japan
}

(Presented 9 June 2016; received 2 June 2016; accepted 7 September 2016; published online 5 October 2016)

\begin{abstract}
We have developed an analysis method to improve the accuracies of electron temperature measurement by employing a fitting technique for the raw Thomson scattering (TS) signals. Least square fitting of the raw TS signals enabled reduction of the error in the electron temperature measurement. We applied the analysis method to a multi-pass (MP) TS system. Because the interval between the MPTS signals is very short, it is difficult to separately analyze each Thomson scattering signal intensity by using the raw signals. We used the fitting method to obtain the original TS scattering signals from the measured raw MPTS signals to obtain the electron temperatures in each pass. Published by AIP Publishing. [http://dx.doi.org/10.1063/1.4963161]
\end{abstract}

\section{INTRODUCTION}

Thomson scattering (TS) diagnostics is one of the most useful methods for measuring electron temperature, $T_{\mathrm{e}}$, and density, $n_{\mathrm{e}}$, in fusion plasmas. ${ }^{1-14}$ For the tandem mirror GAMMA 10/PDX, we developed TS diagnostics for $T_{\mathrm{e}}$ and $n_{\mathrm{e}}$ measurements for single laser and plasma shots. ${ }^{6}$ The value of $n_{\mathrm{e}}\left(\sim 2 \times 10^{18} \mathrm{~m}^{-3}\right)$ in the GAMMA 10/PDX plasma is typically lower than that in other fusion plasmas. ${ }^{15}$ The TS signal processing method is important when the TS signal is weak. We therefore adopted a large solid angle of TS collection optics and high-sensitivity detection systems for the weak TS signals. In a polychromator-type TS system in fusion devices, a high-speed charge-to-digital converter system is usually used to obtain the TS signal intensity. ${ }^{1,2,4}$ Some TS systems use high-speed analog-todigital converters or oscilloscopes to obtain the raw signal waveforms. ${ }^{6-11}$ The output signals of the polychromators in a TS system are normally fitted to a Gaussian function in order to obtain the single-pass TS signal intensities. ${ }^{7-11}$ TS data processing methods for raw TS signals with high-timeresolution data acquisition systems have been developed for electron temperature and density measurements, e.g., MonteCarlo simulation for analyzing TS signals, ${ }^{12}$ maximumlikelihood fitting method for TS fitting data, ${ }^{13}$ and nonGaussian pulse shape fitting method. ${ }^{14}$ Since most of these methods are complex, we used a simple pulse shape fitting method that considers the decay time of the output signal of the polychromator in the TS system. At the plasma edge region, the TS signal intensity is very low, and the signal-to-noise ratio is low, i.e., $\sim 3$. The TS system in GAMMA 10/PDX can

\footnotetext{
Note: Contributed paper, published as part of the Proceedings of the 21st Topical Conference on High-Temperature Plasma Diagnostics, Madison, Wisconsin, USA, June 2016.

a) Author to whom correspondence should be addressed. Electronic mail: ohta_koichi@prc.tsukuba.ac.jp.
}

measure the radial profiles of $T_{\mathrm{e}}$ and $n_{\mathrm{e}}$ at six radial positions of $\mathrm{X}=0, \pm 5, \pm 10$, and $-15 \mathrm{~cm}$. The errors in the measured $T_{\mathrm{e}}$ and $n_{\mathrm{e}}$ are normally about $30 \%$ and $50 \%$, respectively, when using the normal analysis method that involves summing the signal intensity without performing the fitting. To increase the TS signal intensity, multi-pass (MP) TS systems have been developed in some fusion devices. ${ }^{3,16,17}$ We have previously developed an MPTS system with polarization control and image relaying optical systems. ${ }^{18}$

In this study, we developed a TS analysis method with a fitting technique to reduce the error in the electron temperature measurement for raw TS signals. The MPTS system enables high-time-resolution measurements of the electron temperature with calculation of the electron temperature in every pass. Such high-time-resolution TS measurements are required in high-frequency fluctuation plasma experiments, such as Alfvén ion cyclotron (AIC) modes, electron cyclotron heating experiments, and pellet injection experiments.

\section{ELECTRON TEMPERATURE ANALYSIS METHOD}

The GAMMA 10/PDX-TS system consists of a YAG laser (2 J/pulse, pulse width of $10 \mathrm{~ns}$, and repetition rate of $10 \mathrm{~Hz}$ ), large solid angle TS collection optics, and filter-type polychromators. The output signals of the polychromators are lead to high-speed digital oscilloscopes (IWATSU DS5524, $1 \mathrm{GSa} / \mathrm{s}, 200 \mathrm{MHz}$ ). The polychromators consist of the five wavelength interference filters and five silicon avalanche photodiodes (APDs) with preamplifiers. The preamplifier can amplify the input signals 20 times with a high-speed operational amplifier. Figure 1 shows the wavelength sensitivity of the polychromator and the normalized TS spectrum at $T_{\mathrm{e}}=30 \mathrm{eV}$.

In the previous analysis method, the TS signal intensities were obtained by integrating the raw TS signals measured by the oscilloscopes during $75 \mathrm{~ns}$. This method is not suitable 


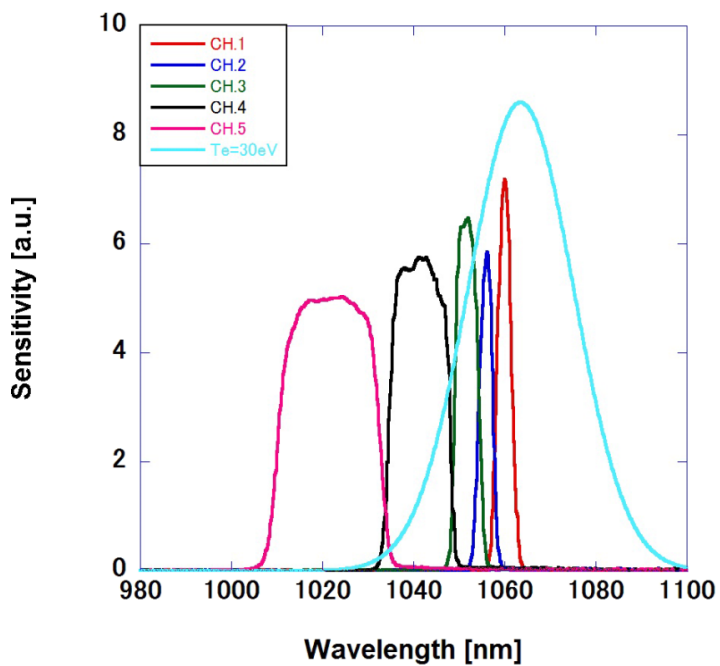

FIG. 1. Wavelength sensitivity of the polychromator and normalized TS spectrum at $T_{\mathrm{e}}=30 \mathrm{eV}$.

for the multi-pass TS signal analysis because the multi-pass TS signals are quite close to each other.

First, we fitted the measured Raman scattering signal that had no background electromagnetic noise and stray light. Setting this signal as the base scattering signal waveform of the output signal of the polychromator is advantageous. Figure 2 shows the measured Raman signal (red solid line) and the fitted waveform (blue dotted line) fitted using the following fitting function. The waveform function $f$ is shown by the discrete convolution of the impulse response $g$ and the scattering signal $h$,

$$
\begin{gathered}
f(t)=\sum_{\tau=0} g(\tau) h(t-\tau), \\
g\left(t, \tau^{\prime}\right)=e^{t / \tau^{\prime}}, \\
h\left(t, \mathrm{~A}, t^{\prime}\right)=\mathrm{A} \cdot \exp \left(-\frac{\left(t-t^{\prime}\right)^{2}}{d}\right) .
\end{gathered}
$$

Here, $\tau$ is a variable, $\tau^{\prime}$ is the time constant of the RC circuit at approximately $20 \mathrm{~ns}, \mathrm{~A}$ is the signal intensity, and $t^{\prime}$ is the start time of the scattering signal. The pulse width $d$ of

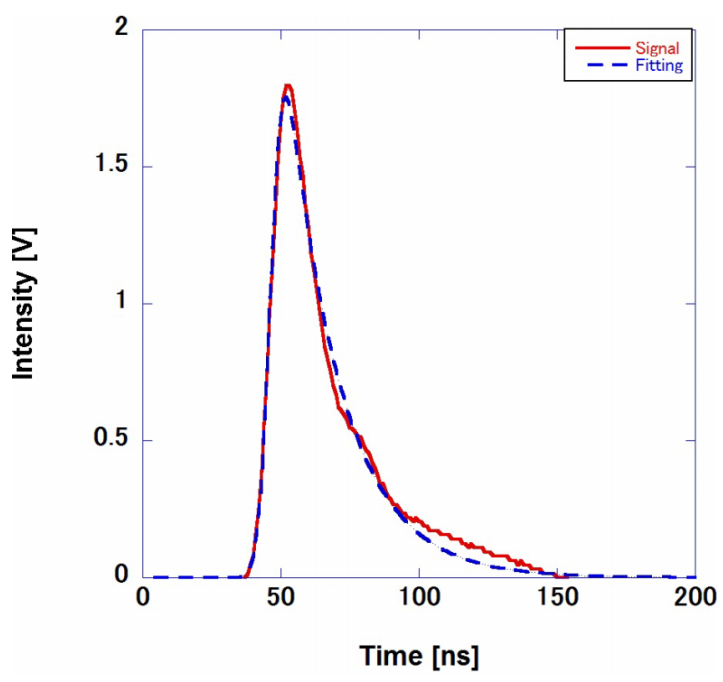

FIG. 2. Fitted waveform and measured Raman signal. scattering signal $h$ is 25 at approximately $10 \mathrm{~ns}$. We use $\tau^{\prime}, \mathrm{A}$, and $t^{\prime}$ as the parameters. The correlation coefficient is $99 \%$.

The chi-square method is used for measuring the electron temperature and error with the signal intensities, background noises, and a lookup table that contains the calculated intensities expected in each channel up to $200 \mathrm{eV}$ at $1 \mathrm{eV}$ intervals. In the conventional method, the signal intensities are analyzed by using the previous analysis method. The measurement accuracies are $34 \pm 4 \mathrm{eV}$ at $\mathrm{X}=0 \mathrm{~cm}$ and $27 \pm 8 \mathrm{eV}$ at $\mathrm{X}=10 \mathrm{~cm}$ with the average of over 70 plasma shots. The error ratios are $13 \%$ and $30 \%$ at $\mathrm{X}=0 \mathrm{~cm}$ and $\mathrm{X}=10 \mathrm{~cm}$, respectively.

Through analysis using the new fitting method, we can obtain the integrated signal intensities from the waveform function $f$. The obtained electron temperatures at $\mathrm{X}=0 \mathrm{~cm}$ and $\mathrm{X}=10 \mathrm{~cm}$ are $37 \pm 4 \mathrm{eV}$ and $27 \pm 6 \mathrm{eV}$, respectively. The chi-square values decrease, and the measurement accuracies are improved from $13 \%$ to $11 \%$ at $\mathrm{X}=0 \mathrm{~cm}$ and $30 \%$ to $22 \%$ at $X=10 \mathrm{~cm}$ in terms of the error ratio. This analysis method has a large effect to the plasma edge region. This new method is useful for overlapping signals, such as the multi-pass TS signals.

\section{ANALYSIS OF MPTS SIGNALS}

We have previously developed an MPTS system that uses polarization optics and an image relay system to increase the TS signal intensity. ${ }^{3,16,17}$ The MP system controls the polarization of the laser beam with a Pockels cell (FastPulse, Q1059P12SG-1064) and a polarizer, and confines the laser beam between the two reflection mirrors. In Fig. 3(a), the MPTS signal and fitted waveform of MPTS signal are shown with the red solid line and blue dotted line, respectively. The signal intensity summed from the 1st-pass to the 10th-pass TS signal is up to about five times larger than the 1st-pass TS signal intensity. The different intervals between the pass signals result from the different distances between the reflection mirror and the plasma in the arrangement of the optics.

The interval between each MPTS signal is too short to analyze the TS signal at each pass using the raw TS signal. We then fitted the 1st-pass TS signal and subtracted its waveform from the MP signal. We repeated this procedure for the next TS pass signal and fit all the signals of each pass to analyze each TS signal intensity. The electron temperatures of the 1st-pass TS signal, TS signals from the 1st to the 6th pass, and from the 1st to the 10th pass are $23 \pm 4 \mathrm{eV}, 22 \pm 2 \mathrm{eV}$, and $22 \pm 3 \mathrm{eV}$, respectively. The $T_{\mathrm{e}}$ calculation using the TS signals from the 1 st to the 6th pass is the most effective for decreasing the measurement error from $17 \%$ to $9 \%$, as compared with the 1st-pass TS signal. The accuracy of the electron temperature measurement is thus improved by using MPTS. We used the new fitting method to obtain the signal intensities of each pass separately and calculated the time dependent $T_{\mathrm{e}}$ in the interval of 20 or 50 ns (Figure 3(b)), which refers to MHz sampling. The errors for the TS signals from the 7th to the 10th pass are large because of the low signal intensities. For the TS signals from the 1 st to the 6 th pass, the errors are about $15 \%$. There is thus a variation in the obtained electron temperatures. However, the electron collision time is about $700 \mathrm{~ns}$ in this 

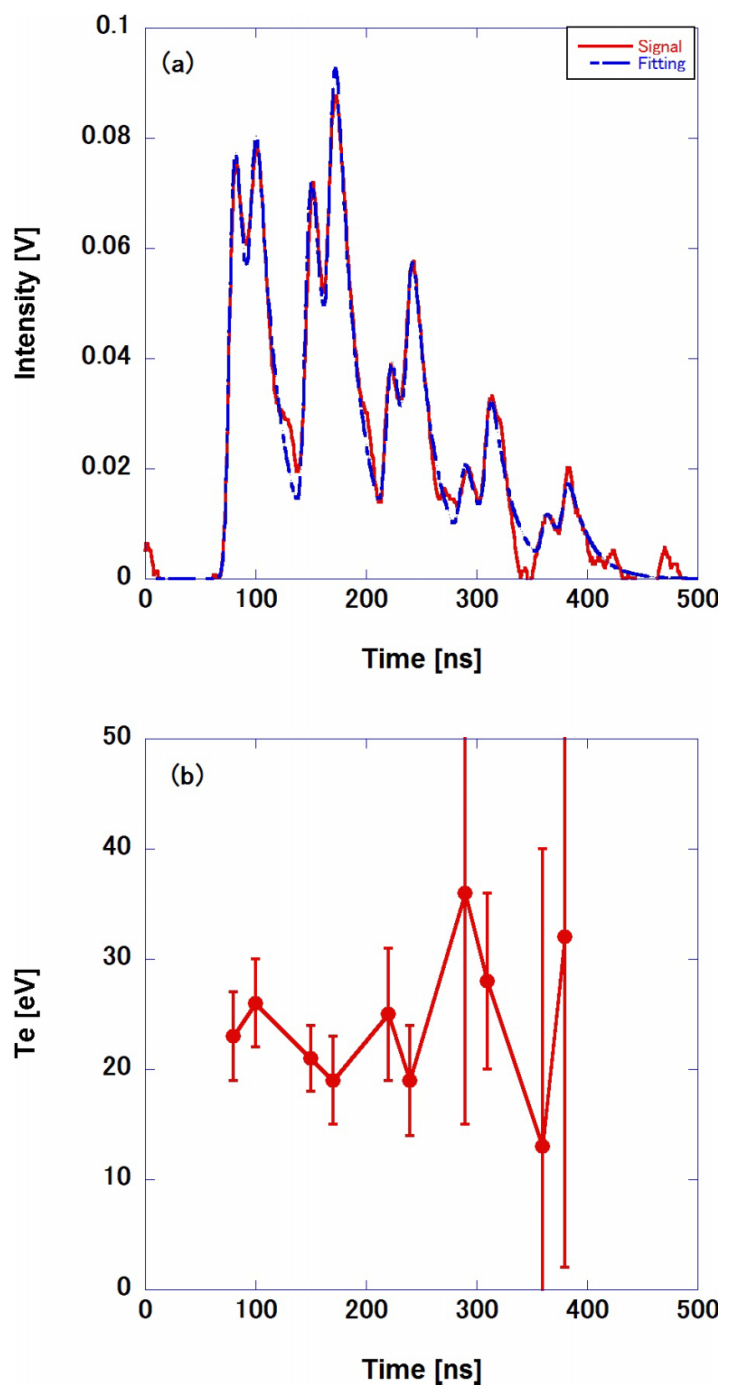

FIG. 3. (a) Fitted waveform and multi-pass TS signal. (b) Time development of the electron temperature.

plasma, and the electron temperature is almost constant during the measured $400 \mathrm{~ns}$. In low temperature plasmas such as divertor plasmas, SMBI (Supersonic Molecular Beam Injection), and pellet-injected plasmas, high time resolved electron temperature measurement is important, and the MPTS system and this analysis method may be used in those cases.
We thus successfully improved the accuracy of the electron temperature measurement by using the fitting method, and we obtained high time-resolved electron temperature measurements of MPTS signals at a high repetition rate.

\section{SUMMARY}

We developed an analysis method by fitting the raw TS signals for obtaining the original signal intensities in order to improve the accuracy of $T_{\mathrm{e}}$ measurements. The errors in the $T_{\mathrm{e}}$ measurements decreased at both the core and the edge region. Moreover, we applied the analysis method to MPTS signals and successfully measured the time-dependent $T_{\mathrm{e}}$ values with $\mathrm{MHz}$ sampling.

\section{ACKNOWLEDGMENTS}

The authors thank the members of the GAMMA 10 group of the University of Tsukuba for their collaboration. This study was conducted with the support and under the auspices of the NIFS Collaborative Research Program, NIFS-KUGM056 and NIFS-KOAH025.

${ }^{1}$ K. Narihara et al., Fusion Eng. Design 34-35, 67 (1997).

${ }^{2}$ K. Narihara, I. Yamada, H. Hayashi, and K. Yamauchi, Rev. Sci. Instrum. 72, 1122 (2001).

${ }^{3}$ M. Tsalas et al., J. Instrum. 7, C03015 (2012).

${ }^{4}$ T. Hatae et al., Rev. Sci. Instrum. 70, 772 (1999).

${ }^{5}$ H. J. van der Meiden et al., Rev. Sci. Instrum. 79, 013505 (2008).

${ }^{6}$ M. Yoshikawa et al., J. Instrum. 8, C10016 (2013).

${ }^{7}$ T. Sumikawa, K. Yamashita, E. Kawamori, K. Narihara, and Y. Ono, Plasma Fusion Res. 2, S1108 (2007).

${ }^{8}$ M. Aftanas, P. Bílková, P. Böhm, V. Weinzettl, J. Stöckel, M. Hron, and R. Pánek, WDS'11 Proceedings of Contributed Papers. Part II (2011), pp. 237-240.

${ }^{9}$ M. Aflanas, Rev. Sci. Instrum. 83, $10 \mathrm{E} 350$ (2012).

${ }^{10}$ T. Yamaguchi et al., Plasma Fusion Res. 5, S2092 (2010).

${ }^{11}$ Z. Qing et al., Plasma Sci. Technol. 12, 144 (2010).

${ }^{12}$ M. Maslov, M. N. A. Beurskens, J. Flanagan, M. Kempenaars, and JET EFDA Contributors, Rev. Sci. Instrum. 83, 096106 (2012).

${ }^{13}$ M. R. Stoneking and D. J. Den Hartog, Rev. Sci. Instrum. 68, 914 (1997).

${ }^{14}$ B. Kurzan, M. Jakobi, H. Murmann, and ASDEX Upgrade Team, Plasma Phys. Controlled Fusion 46, 299-317 (2004).

${ }^{15}$ Y. Nakashima et al., J. Nucl. Mater. 463, 537-540 (2015).

${ }^{16}$ J. Hiratsuka et al., Plasma Fusion Res. 5, 044 (2010).

${ }^{17}$ M. Yu Kantor et al., Plasma Phys. Controlled Fusion 51, 055002 (2009).

${ }^{18}$ M. Yoshikawa et al., J. Instrum. 10, T08003 (2015). 